\title{
A Texton-Based Approach for the Classification of Lung Parenchyma in CT Images
}

\author{
Mehrdad J. Gangeh ${ }^{1}$, Lauge Sørensen ${ }^{2}$, Saher B. Shaker ${ }^{3}$, \\ Mohamed S. Kamel ${ }^{1}$, Marleen de Bruijne', ${ }^{2,4}$ and Marco Loog \\ ${ }^{1}$ Department of Electrical and Computer Engineering, University of Waterloo, Canada \\ \{mgangeh, mkamel\} @pami . uwaterloo.ca \\ ${ }^{2}$ Department of Computer Science, University of Copenhagen, Denmark \\ \{lauges, marleen @ diku. dk \\ ${ }^{3}$ Department of Respiratory Medicine, Gentofte University Hospital, Hellerup, Denmark \\ ${ }^{4}$ Biomedical Imaging Group Rotterdam, Erasmus MC, The Netherlands \\ ${ }^{5}$ Pattern Recognition Laboratory, Delft University of Technology, The Netherlands \\ m.loog@tudelft.nl
}

\begin{abstract}
In this paper, a texton-based classification system based on raw pixel representation along with a support vector machine with radial basis function kernel is proposed for the classification of emphysema in computed tomography images of the lung. The proposed approach is tested on 168 annotated regions of interest consisting of normal tissue, centrilobular emphysema, and paraseptal emphysema. The results show the superiority of the proposed approach to common techniques in the literature including moments of the histogram of filter responses based on Gaussian derivatives. The performance of the proposed system, with an accuracy of $96.43 \%$, also slightly improves over a recently proposed approach based on local binary patterns.
\end{abstract}

\section{Introduction}

Computerized quantitative analysis in pulmonary computed tomography (CT) images is a vital tool in the analysis of chronic obstructive pulmonary disease (COPD). The disease is projected to become the fifth most burdening disease worldwide by 2020 [1]. COPD is a chronic lung disease characterized by limitation of airflow, and it comprises two components: small airway disease and emphysema, which is characterized by gradual loss of lung tissue.

Current methods for the diagnosis and quantification of COPD suffer from several limitations. Common computerized methods on CT images do not use all the information available in a CT image. For example, the relative area of emphysema below a threshold (RA) [2] considers only independent pixel intensity values and relies on a hand-picked parameter, the threshold. The primary diagnostic tool for COPD is spirometry by which various pulmonary function tests (PFTs) are performed [1]. These are cheap and fast to acquire, but they have a low sensitivity to early stages of COPD.

This work focuses on improving the assessment of emphysema in CT images. Emphysema lesions, or bullae, are visible in CT images as areas of abnormally low attenuation values close to that of air, and it is often classified into three subtypes [3]. 
These subtypes are the following: centrilobular emphysema (CLE), defined as multiple small low-attenuation areas; paraseptal emphysema (PSE), defined as multiple low-attenuation areas in a single layer along the pleura often surrounded by interlobular septa that is visible as thin white walls; and panlobular emphysema (PLE), defined as a low-attenuation lung with fewer and smaller pulmonary vessels.

As the texture of lung tissue is affected by the type of disease, texture analysis can be used for quantitative assessment of different subtypes of emphysema. Classifying emphysema in CT images of the lung using texture features was first introduced in [4]. Since then, various features have been used for the classification of emphysema and other disorders in lung CT images including moments of histograms computed on the outputs of filter banks consisting of Gaussian derivatives [5], measures on graylevel co-occurrence matrices (GLCM), measures on gray-level run-length matrices (GLRLM), and moments of the attenuation histogram [4, 6, 7].

Recently, it was shown that small-sized local operators like local binary patterns (LBP) [8] and the patch representation of small local neighborhood in texton-based approaches [9] yield excellent texture classification performance on standard texture databases. It should be noted here that small-sized local operators are desirable in situations where the region of interest (ROI) is rather small, which is often the case in texture analysis in medical imaging, where pathology can be localized in small areas. This is because of two reasons: first, convolution with large support filter banks suffers from boundary effects; second, more patches can be extracted using small-sized local operators that makes the estimation of image statistics more reliable [9].

In this paper, we propose to use small patch representation in texton-based approaches along with support vector machines (SVMs) for the classification of emphysema in CT images of the lung. To our knowledge, this technique has never been used for the classification of CT lung images.

The effectiveness of small-sized local operators in medical imaging is shown in [10] using LBP texture features and $k$-NN classifier with similar results to filter bank approaches based on Gaussian derivatives. In this work, we also show that textonbased approaches using a SVM with radial basis function (RBF) kernel produces better results than common filter bank approaches and slightly better results than LBP, which can be considered as the state of the art in emphysema classification [10].

\section{Texton-Based Texture Classification}

In this section, the principle of texton-based texture classification is reviewed $[9,11,12]$. This approach is independent of the representation used to describe local image information, i.e., it could be raw pixel representation, outputs of filter banks convolved with the patches, or even more complex representations. The texton-based approach can be divided into three stages: 1) construction of a codebook of textons using a clustering algorithm such as $k$-means, 2) learning texton histograms from the training set, and 3) classification of the test set by finding the histogram of textons in the test set and comparing to those found during stage two to find the nearest pattern. These three steps are explained in the remaining of this section. 


\subsection{Construction of Texton Codebook}

To construct the texton codebook, small-sized local patches are randomly extracted from each image in the training set. These small patches are then aggregated over all images in a class and clustered using an unsupervised algorithm such as $k$-means. Obtained cluster centers form a dictionary that represents the class of textures used. It is saved as the codebook to be used in the next stage. Fig. 1 displays sample images of lung CT ROIs used in this paper and the codebook dictionary computed over all ROIs using the texton size of $7 \times 7$ pixels and $k=40$ in $k$-means.

\subsection{Learning the Model}

The next stage is to find the features (learn the model) using the images in the training set. To this end, these steps are followed: first, extract small patches of the same size as the previous stage by sliding a window over each training image in a class. Second, find the distance between each patch to all textons in the dictionary to find the closest match. Third, update a histogram of textons accordingly for each image based on the closest match found. This yield a histogram for each image in the training set, which is used as the features representing that image after normalization. These features are used for training a classifier such as SVM. Fig. 2 illustrates the construction of the codebook and learning the model in a texton-based classification system.

\subsection{Classification}

To classify a test image, the same steps as in the learning stage are followed to find the features for the test image. This includes extraction of small patches from each test image in a class, finding the closest match to these patches from the dictionary, and computing the normalized histogram of obtained closest textons to define a feature vector for the image. The trained classifier in the learning stage is used to find the class of the test image.

Both $k$-NN and SVM are tested in this paper for the classification of texton-based features. In SVM, a RBF kernel as given in (1) is used as it is recommended as the first kernel choice in [13]. In (1), $\gamma$ is the kernel width and $\mathbf{x}_{i}$ and $\mathbf{x}_{\mathbf{j}}$ are two sample patterns.

$$
K\left(\mathbf{x}_{i}, \mathbf{x}_{j}\right)=e^{-\gamma\left\|\mathbf{x}_{i}-\mathbf{x}_{j}\right\|^{2}} .
$$

\section{Experimental Setup}

Data Preparation. The data used for the experiments is the same as in [10], which is collected from a set of thin-slice CT images of the thorax from an exploratory study carried out at the Department of Respiratory Medicine, Gentofte University Hospital, Denmark [14]. The slices were reconstructed using a high spatial resolution (bone) algorithm. Each subject was scanned in the upper, middle, and lower lung, resulting in three $1.25 \mathrm{~mm}$ thick slices with a resolution of $0.78 \times 0.78 \mathrm{~mm}$ per subject.

The leading pattern in $75 \mathrm{CT}$ slices from 25 subjects, 8 healthy non-smokers, 4 smokers without COPD, and 13 smokers diagnosed with moderate or severe COPD 

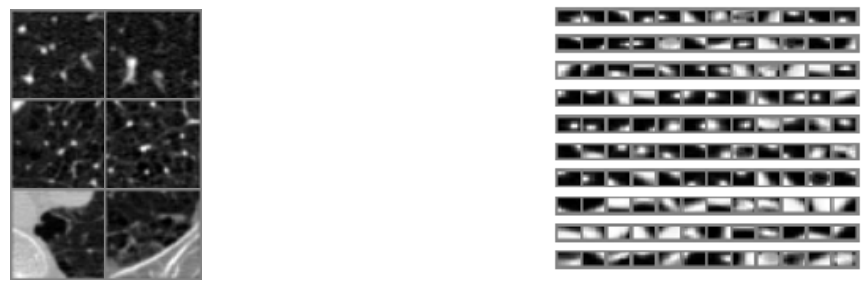

Fig. 1. Sample ROIs of size $50 \times 50$ pixels (left) in three classes, i.e., normal lung (top left row), CLE (middle left row), and PSE (bottom left row). The constructed codebook using texton sizes of $7 \times 7$ pixels and $\mathrm{k}=40$ in $k$-means $(r i g h t)$.
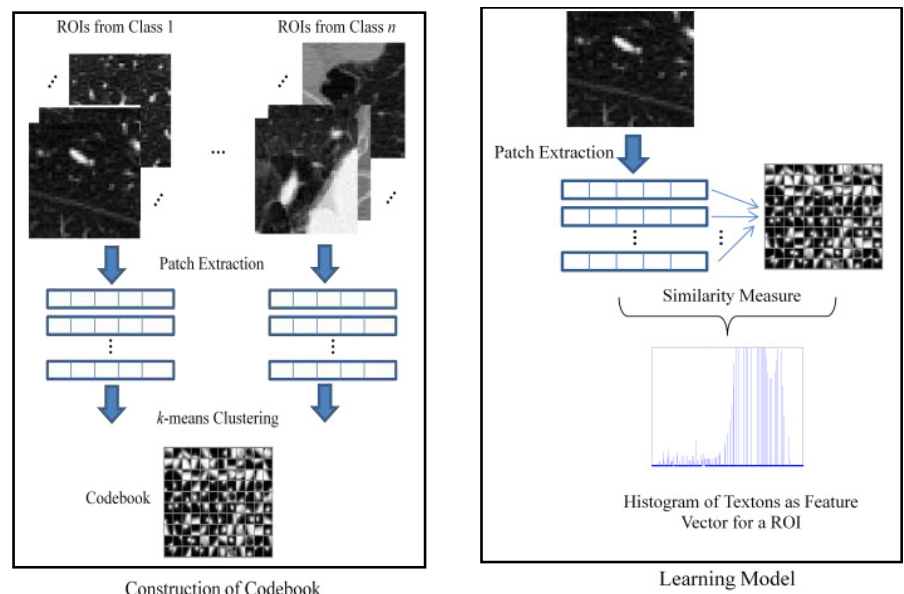

Fig. 2. The illustration of different stages of a texton-based texture classification system: the generation of texton codebooks using $k$-means clustering (left) and the generation of features by computing the texton histograms of training set (right).

according to PFTs [1], was visually assessed by an experienced chest radiologist and a CT experienced pulmonologist independently. The leading pattern could either be normal tissue (NT), CLE, PSE, or PLE, in each of the slices, and consensus readings were obtained in all cases of disagreement. 168 non-overlapping ROIs of size $50 \times 50$ pixels were subsequently annotated in the slices representing the three classes: NT (59 ROIs), CLE (50 ROIs), and PSE (59 ROIs). The NT ROIs were annotated in the nonsmokers and the CLE and PSE ROIs were annotated in the two smokers' subject classes, within the area(s) of the leading pattern. PLE was excluded due to very few cases in the data set (only 2 out of 20 subjects diagnosed with COPD).

Computation of Texton-Based Features. For the construction of the codebook, 500 random patches are extracted from each ROI in each class. Patch sizes of $3 \times 3$ to $8 \times$ 8 pixels are used in the experiments. No filter banks are applied and raw pixel representation is used instead. Since in CT images, the mean of the intensity in the images 
indicate a physical property of the tissue displayed, it should not be removed. The patches extracted from different ROIs of each class are clustered using $k$-means to find the codebook that represents a class. Two different values of $k$, i.e., $k=10$ and $k$ $=40$ are tested in the experiments leading to $3 \times 10=30$ or $3 \times 40=120$ ( 3 is the number of classes) textons in the codebook, respectively (refer to Fig. 1). After construction of texton codebook, the texton frequency histograms of the ROIs are computed to find the model. In this stage, small overlapping patches with the same size as what was used in the clustering stage are systematically extracted from each ROI. As in the clustering stage, no filter bank is used and raw pixel representation is considered. Euclidean distance between the resulting textons (collection of small patches) and the textons in the codebook is computed in order to identify the most similar texton in the codebook and the corresponding histogram of textons is updated accordingly. The histograms are normalized and used as the features.

Classifier and Evaluation. Both $k-\mathrm{NN}$ and SVM are used in the experiments. The crucial issue in using SVMs is finding a suitable kernel and the optimum trade-off parameter $C$. RBF kernel is used and the optimum kernel width, i.e., $\gamma$ in (1) and $C$ are found by a grid search on the training set at each specific texton size and $k$ value (in $k$ means) used in the experiments. This grid search is performed by leave-one-subjectout on the training set. The computation of the texton codebook is performed each time on the training set, excluding the validation set. The proposed texton-based classification system using SVM as classifier with RBF kernel and optimal $C$ and $\gamma$ is evaluated using leave-one-subject-out. This means that, at each trial, all ROIs from one patient are held out as the test set and the remaining ROIs as the training set.

\section{Results and Discussions}

In this section, we first present the results for the proposed texton-based texture classification system using SVM as classifier with the parameters chosen as explained in previous section. Then comparison with other techniques is provided.

After finding the optimal $C$ and $\gamma$ at each texton size and $k$ value (of $k$-means), the accuracy of the classification system is evaluated using leave-one-subject-out for the particular texton size and $k$. The results are shown in Table 1 for various texton sizes and two different values of $k$ in $k$-means. It can be seen from these results that using $k$ $=40$ in $k$-means improves the performance of the classification system over $k=10$. The best result is obtained at the texton size of $4 \times 4$ pixels with $k=40$. It can be observed from the results that the accuracy of classification system decreases with increasing texton size. This can be because increasing the texton size leads to a higher dimensional space in $k$-means, requiring more data for reliable clustering. Also, fewer patches can be extracted from the ROIs at higher texton sizes that may degrade the estimation of model as explained in Section 2.2.

Comparison with Other Techniques. The first comparison is made between textonbased classification system using SVM and $k$-NN classifiers. The optimal parameter $k$ of the $k$-NN classifier is found using a validation set in the training set in a similar way as the grid search performed for the parameters in the SVM classifier. The results are shown in Table 1, and as can be seen, the SVM classifier performs much better 
Table 1. The results of texton-based classification system on CT images of lung used in this paper for $k=10$ and 40 and various texton sizes using SVM and $k$-NN classifiers.

\begin{tabular}{|c|c|c|c|c|}
\hline Texton Size & $\begin{array}{c}\text { SVM Accuracy } \\
\left(\boldsymbol{k}^{\text {(a) }}=\mathbf{1 0}\right)\end{array}$ & $\begin{array}{c}\text { SVM Accuracy } \\
\left(\boldsymbol{k}^{(\mathbf{a})}=\mathbf{4 0}\right)\end{array}$ & $\begin{array}{c}\boldsymbol{k} \text {-NN Accuracy } \\
\left(\boldsymbol{k}^{\text {(a) }}=\mathbf{1 0}\right)\end{array}$ & $\begin{array}{c}\boldsymbol{k} \text {-NN Accuracy } \\
\left(\boldsymbol{k}^{\text {(a) }}=\mathbf{4 0}\right)\end{array}$ \\
\hline $3 \times 3$ & $\mathbf{9 4 . 0 5}$ & 95.24 & $\mathbf{9 0 . 4 8}$ & 88.1 \\
\hline $4 \times 4$ & 93.45 & $\mathbf{9 6 . 4 3}$ & 86.31 & 86.9 \\
\hline $5 \times 5$ & 92.86 & 95.83 & 85.12 & 88.1 \\
\hline $6 \times 6$ & 92.26 & 94.05 & 82.74 & $\mathbf{9 0 . 4 8}$ \\
\hline $7 \times 7$ & 91.67 & 90.48 & 83.33 & 89.29 \\
\hline $8 \times 8$ & 88.10 & 92.86 & 82.74 & 89.88 \\
\hline
\end{tabular}

(a) $k$ here refers to the $k$ in $k$-means clustering not the $k$ in $k$-NN classifier.

than the $k$-NN classifier. The superiority of SVM over $k$-NN was also reported on standard texture databases such as Columbia-Utrecht (CUReT) database [15].

The second comparison is made between the proposed texton-based classification system using SVM as classifier and the results published in [10]. Since the same data as in [10] is used in our experiments, the results are directly comparable. In [10], the results are provided for the following approaches:

1) GFB1 (Gaussian filter bank 1): using the moments of histogram computed on the outputs of convolved Gaussian filter banks with four rotation invariant filters obtained from linear combination of Gaussian derivatives at five scales.

2) ICR (intensity, co-occurrence, and run-length): the feature vector consists of the first four moments of the intensity histogram; the contrast, correlation, energy, entropy, and homogeneity computed on GLCM; and short-run emphasis, long-run emphasis, gray-level; nonuniformity, run-length nonuniformity and run percentage computed on GLRLM.

3) INT: intensity histograms.

4) GFB2: similar to GFB1 but instead of using moments of histograms, the histograms themselves are used. Hence they are richer in information.

5) LBP1 (local binary pattern 1): basic rotation invariant LBP histograms.

6) LBP2: joint 2D LBP and intensity histograms.

For more information on each method the reader is urged to refer to [10] and the references therein. The results based on the above techniques are provided in Table 2 along with the best result obtained from the proposed approach based on texton signatures with SVM classifier.

Table 2. The comparison between the best results obtained from the proposed approach and the results of other techniques on the same data (left); the confusion matrix of LBP2 (middle) and texton-based approach with $k=40$, texton size of $4 \times 4$ pixels, and SVM classifier (right).

\begin{tabular}{|c|c|c|c|c|c|c|c|c|c|}
\hline $\begin{array}{l}\text { Technique } \\
\text { GFB1 }\end{array}$ & Accuracy & & \multicolumn{3}{|c|}{ Estimated Labels } & & \multicolumn{3}{|c|}{ Estimated Labels } \\
\hline ICR & 89.3 & True Labels & NT & CLE & PSE & True Labels & NT & CLE & PSE \\
\hline $\begin{array}{l}\text { INT } \\
\text { GFB2 }\end{array}$ & $\begin{array}{l}87.5 \\
94.0\end{array}$ & NT & 55 & 0 & 4 & NT & 58 & 0 & 1 \\
\hline LBP1 & 79.2 & CLE & 1 & 49 & 0 & CLE & 3 & 47 & 0 \\
\hline $\begin{array}{l}\text { LBP2 } \\
\text { Texton-based }\end{array}$ & $\begin{array}{l}95.2 \\
96.4\end{array}$ & PSE & 2 & 1 & 56 & PSE & 2 & 0 & 57 \\
\hline
\end{tabular}


The confusion matrix for the best results in [10], i.e., the results of LBP2, and our best results are provided in Table 2 . The proposed approach attains performance better than LBP2 though McNemar's test does not indicate the difference to be significant $(p=0.75)$. The specificity of texton-based and LBP2 approaches are $98.31 \%$ and $93.33 \%$, while their sensitivity are $95.41 \%$ and $97.25 \%$, respectively (when comparing NT versus CLE and PSE).

\section{Conclusion}

In this paper, a texton-based texture classification system using a SVM with RBF kernel is proposed for the classification of emphysema in CT images of the lung. It is shown that the proposed approach performs significantly better than common approaches based on moments of histograms of filter bank responses using Gaussian derivatives and slightly improve the performance over a recently proposed approach based on LBPs. LBP operators are, by design, invariant to monotonic intensity transformations. While this is desirable in some applications, in the classification of Lung CT images, the mean of intensity is important and this explains the poor performance of LBP1 in Table 2 as it discards the mean of intensity in the ROIs. Due to this drawback of LBPs, in [10], the joint intensity and LBP histograms are considered (LBP2). This improves the performance of the LBPs in this application at the cost of adding to the complexity of the approach. The textonbased approach does not suffer from this problem as it is not invariant to intensity transformations. It is also shown that using SVM in the proposed approach yields higher accuracy than a $k$-NN classifier.

As the state of the art in examination of lung is volumetric chest CT scans, one may wonder whether texton-based approach can be extended to $3 \mathrm{D}$ data. As the computation of texton signatures is fast especially when raw pixel representation is used, we expect that the approach can easily be extended to 3D data. The main obstacle might be the computational cost for optimizing the SVM parameters in a grid search that can be reduced by using an $m$-fold cross-validation at patient level instead of leave-one-subject-out used in our experiments.

In future work, combining the classification outputs at different texton sizes using multiple classifier systems (MCS) will be investigated for possible improvement of the results. This improvement is expected if the misclassification is done on different ROIs in different texton sizes such that the MCS yield better results due to the diversity of the classifiers [16].

Acknowledgments. This work was partly funded by the Natural Sciences and Engineering Research Council (NSERC) of Canada under Canada Graduate Scholarship (CGS D3-378361-2009), the Danish Council for Strategic Research under the Programme Commission for Nanoscience and Technology, Biotechnology and IT, the Netherlands Organization for Scientific Research (NWO), and AstraZeneca, Lund, Sweden. The first author would also like to thank Laurens van der Maaten from Delft University of Technology, the Netherlands for useful discussions on texton-based approach in texture classification. 


\section{References}

1. Rabe, K.F., Hurd, S., Anzueto, A., Barnes, P.J., Buist, S.A., Calverley, P., Fukuchi, Y., Jenkins, C., Rodriguez-Roisin, R., van Weel, C., Zielinski, J.: Global Strategy for the Diagnosis, Management, and Prevention of Chronic Obstructive Pulmonary Disease: GOLD Executive Summary. Am. J. Respir. Crit. Care Med. 176(6), 532-555 (2007)

2. Müller, N.L., Staples, C.A., Miller, R.R., Abboud, R.T.: Density Mask-An Objective Method to Quantitate Emphysema Using Computed Tomography. Chest 94(4), 782-787 (1988)

3. Webb, W.R., Müller, N., Naidich, D.: High-Resolution CT of the Lung, 3rd edn. Lippincott Williams \& Wilkins (2001)

4. Uppaluri, R., Mitsa, T., Sonka, M., Hoffman, E.A., McLennan, G.: Quantification of Pulmonary Emphysema from Lung Computed Tomography Images. Amer. J. Respir. Crit. Care Med. 156(1), 248-254 (1997)

5. Sluimer, I.C., Prokop, M., Hartmann, I., van Ginneken, B.: Automated Classification of Hyperlucency, Fibrosis, Ground Glass, Solid, and Focal Lesions in High-Resolution CT of the Lung. Medical Physics 33(7), 2610-2620 (2006)

6. Chabat, F., Yang, G.Z., Hansell, D.M.: Obstructive Lung Diseases: Texture Classification for Differentiation at CT. Radiology 228(3), 871-877 (2003)

7. Xu, Y., Sonka, M., McLennan, G., Guo, J., Hoffman, E.A.: MDCT-based 3-D Texture Classification of Emphysema and Early Smoking Related Lung Pathologies. IEEE Trans. Med. Imag. 25(4), 464-475 (2006)

8. Ojala, T., Pietikainen, M., Maenpaa, T.: Multiresolution Gray-Scale and Rotation Invariant Texture Classification with Local Binary Patterns. IEEE Trans. on PAMI 24(7), 971-987 (2002)

9. Varma, M., Zisserman, A.: A Statistical Approach to Material Classification Using Image Patch Exemplars. IEEE Trans. on PAMI 31(11), 2032-2047 (2009)

10. Sørensen, L., Shaker, S.B., de Bruijne, M.: Quantitative Analysis of Pulmonary Emphysema Using Local Binary Patterns. IEEE Trans. Med. Imag. 29(2), 559-569 (2010)

11. Leung, T., Malik, J.: Representing and Recognizing the Visual Appearance of Materials Using Three-Dimensional Textons. Int'l. J. Computer Vision 43(1), 29-44 (2001)

12. Varma, M., Zisserman, A.: A Statistical Approach to Texture Classification from Single Images. International Journal of Computer Vision: Special Issue on Texture Analysis and Synthesis 62(1-2), 61-81 (2005)

13. Fan, R.E., Chen, P.H., Lin, C.J.: Working Set Selection Using the Second Order Information for Training SVM. Journal of Mach. Learning Research 6, 1889-1918 (2005)

14. Shaker, S.B., von Wachenfeldt, K.A., Larsson, S., Mile, I., Persdotter, S., Dahlbäck, M., Broberg, P., Stoel, B., Bach, K.S., Hestad, M., Fehniger, T.E., Dirksen, A.: Identification of Patients with Chronic Obstructive Pulmonary Disease (COPD) by Measurement of Plasma Biomarkers. The Clinical Respiratory Journal 2(1), 17-25 (2008)

15. Caputo, B., Hayman, E., Fritz, M., Eklundh, J.O.: Classifying Materials in the Real World. Image and Vision Computing 28(1), 150-163 (2010)

16. Kuncheva, L.I.: Combining Pattern Classifiers Methods and Algorithms. John Wiley \& Sons, New Jersey (2004) 